\title{
Infección por Nocardia cyriacigeorgica en paciente con infección por VIH en etapa SIDA
}

\section{Nocardia cyriacigeorgica infection in AIDS patient}

\author{
Catalina Gutiérrez', Alejandra Céspedes², Paulette Legarraga³, Pedro Morales², Leonardo Chanqueo ${ }^{1,2}$
}

'Servicio de Medicina Interna, Unidad de Infectología adultos, Hospital San Juan de Dios. Santiago, Chile.

${ }^{2}$ Laboratorio Clínico Hospital San Juan de Dios. Santiago, Chile.

${ }^{3}$ Laboratorio Hospital Clínico Universidad Católica. Santiago, Chile.

Conflictos de interés: Catalina Gutiérrez Ruiz declara participación Red Chilena de Micología 2016-2018.

Financiamiento: ninguno.

Recibido: 11 de julio de 2019 / Aceptado: 30 de mayo de 2020

\section{Resumen}

La nocardiosis es una infección localizada o sistémica que afecta principalmente a pacientes inmunocomprometidos, siendo la localización pulmonar el sitio más frecuente. La transmisión proviene principalmente de la inhalación de esporas o mediante la inoculación directa en la piel y mucosa ocular. Se han descrito más de 90 especies de Nocardia, de éstas más de la mitad son reconocidas como patógenos en humanos. Las especies de importancia médica más conocidas son Nocardia farcinica, Nocardia abscessus, Nocardia nova y Nocardia brasiliensis. En Chile hay casos publicados de infección por Nocardia asteroides y $N$. farcinica. Nocardia cyriacigeorgica se considera una especie emergente, no habiendo casos descritos previamente en nuestro país. Presentamos el caso clínico de una nocardiosis pulmonar en un paciente con síndrome de inmunodeficiencia adquirida (SIDA).

Palabras clave: Nocardia spp.; infecciones en inmunocomprometidos; VIH en etapa SIDA.

\section{Introducción}

L a nocardiosis es una infección localizada o sistémica que afecta principalmente a pacientes inmunocomprometidos. Está descrita en individuos con infección por VIH en etapa SIDA, en pacientes con neoplasias, sometidos a trasplante de órganos sólidos y con uso de corticoesteroides ${ }^{1}$.

El género Nocardia spp., perteneciente al grupo Actinomycetes, que también incluye Mycobacterium, Corynebacterium, Gordona y Tsukamurella ${ }^{2}$; corresponden a bacilos grampositivos aeróbicos.

\begin{abstract}
Nocardiosis is a localized or systemic infection that mainly affects immunocompromised patients, with pulmonary localization being the most frequent site. The transmission comes mainly from the inhalation of spores or by direct inoculation into the skin and ocular mucosa. More than 90 species of nocardia are described, of which more than half are recognized as pathogens in humans. The best known species of medical importance are Nocardia farcinica, Nocardia abscessus, Nocardia nova y Nocardia brasiliensis. In Chile, there have been published cases of Nocardia asteroides and Nocardia farcinica infections. Nocardia cyriacigeorgica is considered an emerging species, there being no cases previously described in our country. We present a clinical case of pulmonary nocardiosis in an acquired immunodeficiency syndrome (AIDS) patient.

Keywords: Nocardia, immunocompromised infections, AIDS.
\end{abstract}

Existen más de 90 especies descritas, siendo las más frecuentes y de importancia médica Nocardia farcinica, Nocardia abscessus, Nocardia nova y Nocardia brasiliensis s.4. $^{3,4}$.

Nocardia cyriacigeorgica fue descrita en el año $2001^{5}$, representando en algunas series, la especie más frecuentemente aislada ${ }^{6}$; esto se debería a que estudios recientes han demostrado que $N$. cyriacigeorgica era previamente identificada como Nocardia asteroides, con un patrón de susceptibilidad específico (grupo VI). Actualmente $N$. asteroides se describe en una baja proporción de muestras clínicas ${ }^{3}$.

La afectación más frecuente es pulmonar, debido a que la inhalación 
es la principal vía de exposición bacteriana. Se manifiesta con la aparición de masas, infiltrados, consolidaciones, cavidades, nódulos pulmonares o derrame pleural ${ }^{7,8}$. También se ha descrito el compromiso de sistema nervioso central, de piel y celular subcutáneo. La mortalidad es variable y puede llegar a $50 \%$ en casos de infección diseminada. Dentro de los diagnósticos diferenciales, debe descartarse otras bacterias oportunistas, micobacterias, hongos y neoplasias.

Presentamos el caso clínico de un paciente con síndrome de inmunodeficiencia adquirida (SIDA), severamente inmunocomprometido, que presentó un compromiso pulmonar y un absceso cervical por $N$. cyriacigeorgica.

\section{Caso clínico}

Varón de 39 años de edad, con antecedentes de consumo de drogas (marihuana y cocaína al menos dos veces por semana). Se había realizado el diagnóstico de síndrome de inmunodeficiencia adquirida (SIDA) el año 2014, confirmado por el Instituto de Salud Pública de Chile en relación a una candidiasis orofaríngea. De sus exámenes de ingreso destacaba un recuento de linfocitos CD4 de 16 céls $/ \mathrm{mm}^{3}$ y una carga viral de VIH (CV) 223.148 copias $/ \mathrm{ml}$ (rango intervalo cuantitativo lineal: 40 a 10.000.000 copias/ml de plasma, método ABBOTT Real Time HIV-1).

El estudio de coinfecciones fue negativo para hepatitis B (AgsHB negativo), hepatitis $\mathrm{C}$, enfermedad de Chagas y sífilis (VDRL no reactivo). La IgG para Toxoplasma fue positiva en $650 \mathrm{UI} / \mathrm{ml}$.

Inició terapia anti-retroviral (TARV) con zidovudina/ lamivudina y efavirenz, junto con profilaxis con cotrimoxazol $800 \mathrm{mg}$ al día y azitromicina 1 gramo semanal, con buena respuesta clínica. A los cuatro meses de iniciada la TARV, se constató una CV de VIH indetectable (LDL: el resultado es más bajo que el límite inferior de cuantificación $<40$ copias $/ \mathrm{ml}$ de plasma) y recuento de linfocitos CD4 69 céls $/ \mathrm{mm}^{3}$. Sin embargo, el paciente abandonó TARV y controles.

Reingresó a los dos años, encontrándose asintomático; de sus exámenes destacaban un recuento de linfocitos CD4 3 céls $/ \mathrm{mm}^{3}$ y una CV de VIH 390.490 copias $/ \mathrm{ml}$, PPD: $0 \mathrm{~mm}$. La radiografía de tórax presentaba imágenes cicatriciales en LSD (Figura 1). Reinició TARV con tenofovir/emtricitabina y atazanavir/ritonavir más profilaxis con cotrimoxazol y azitromicina; sin embargo, volvió a abandonar la terapia.

Reconsultó tres meses después por un cuadro de un mes de evolución caracterizado por tos con expectoración purulenta, fiebre y compromiso del estado general. A su ingreso al hospital destacaron en los exámenes de laboratorio un recuento de leucocitos $9800 \times \mathrm{mm}^{3}, 74 \%$ neutrófilos, VHS $104 \mathrm{~mm} / \mathrm{h}$ y una proteína C reactiva de
$155 \mathrm{mg} / \mathrm{L}$; la radiografía de tórax demostró una consolidación pulmonar en el lóbulo superior derecho (LSD) (Figura 2).

Se estudió con hemocultivos automatizados que fueron negativos, tinción de Gram y cultivo de expectoración con desarrollo de flora microbiana comensal, panel molecular para patógenos respiratorios positivo para adenovirus y rinovirus. La fibrobroncoscopía con lavado broncoalveolar mostró un recuento de leucocitos 150 céls $/ \mathrm{mm}^{3}$, con

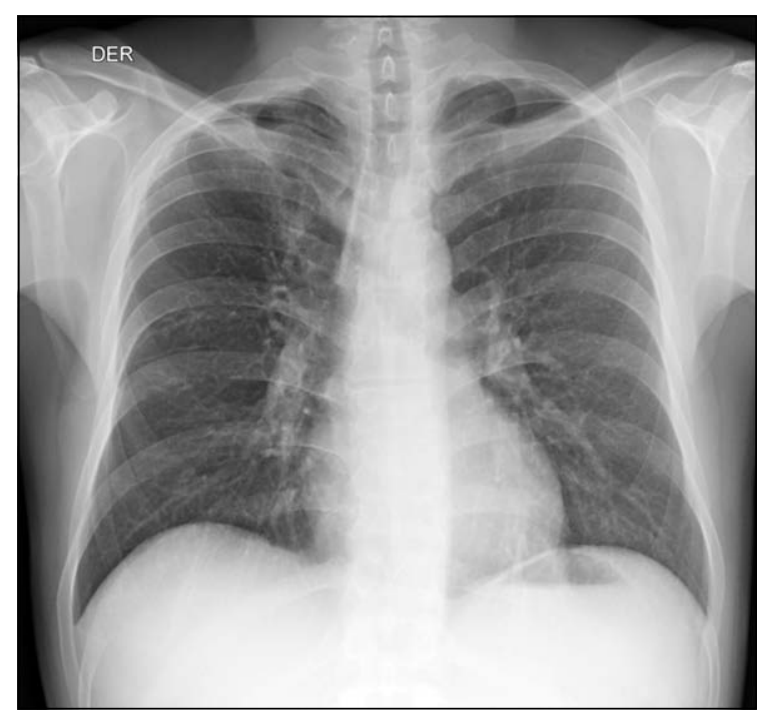

Figura 1. Radiografía de tórax anteroposterior: campos pulmonares de expansión conservada. Con imágenes cicatriciales en el LSD. Mediastino de contornos regulares.

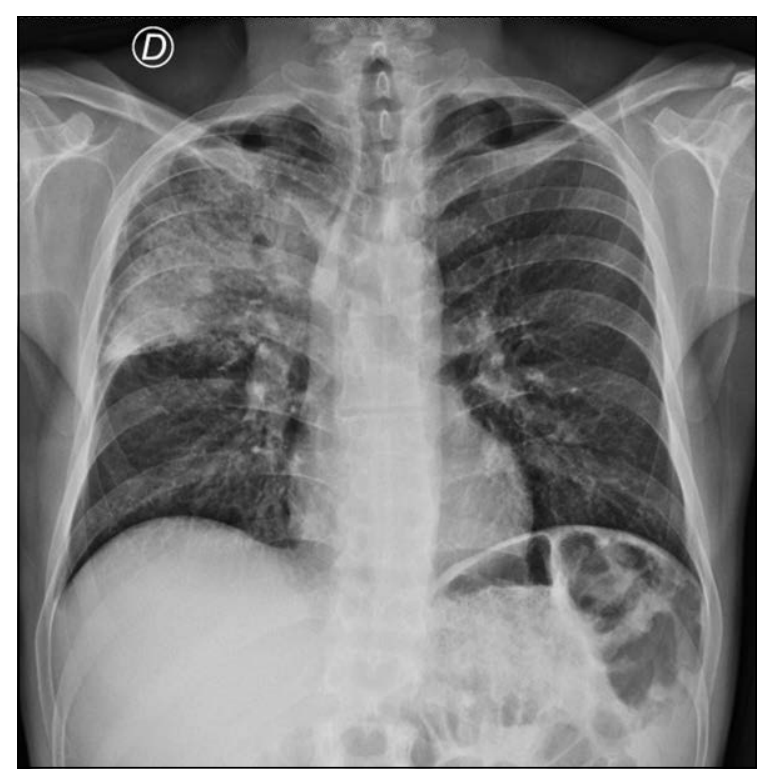

Figura 2. Radiografía de tórax anteroposterior muestra opacidad parenquimatosa que compromete extensamente LSD, y parcialmente la base del campo pulmonar izquierdo. 
$5 \%$ linfocitos y $95 \%$ macrófagos. El cultivo corriente fue negativo. Las baciloscopías, cultivo de micobacterias en medio líquido (MGIT) y en medio sólido (LowesteinJensen) a los 30 y 60 días fueron todos negativos.

Reinició TARV con tenofovir/emtricitabina más atazanavir/ritonavir y profilaxis con cotrimoxazol y azitromicina. Sin embargo, persistió con tos y baja de peso

Figura 3. Tomografía axial de cuello y tórax con una consolidación pulmonar excavada en el lóbulo superior derecho asociado a múltiples adenopatías mediastínicas, algunas de ellas con centro hipo-denso. Además se observa una masa, probablemente adenopática con centro necrótico en la región cervical basal derecha y axilar ipsilateral. A: ventana pulmonar; B: ventana mediastínica.

Figura 4. Tinción de Kinyoun positiva de muestra de LBA donde se observan bacilos largos ramificados ácido alcohol resistentes (Foto original de Laboratorio Microbiología Hospital San Juan de Dios).
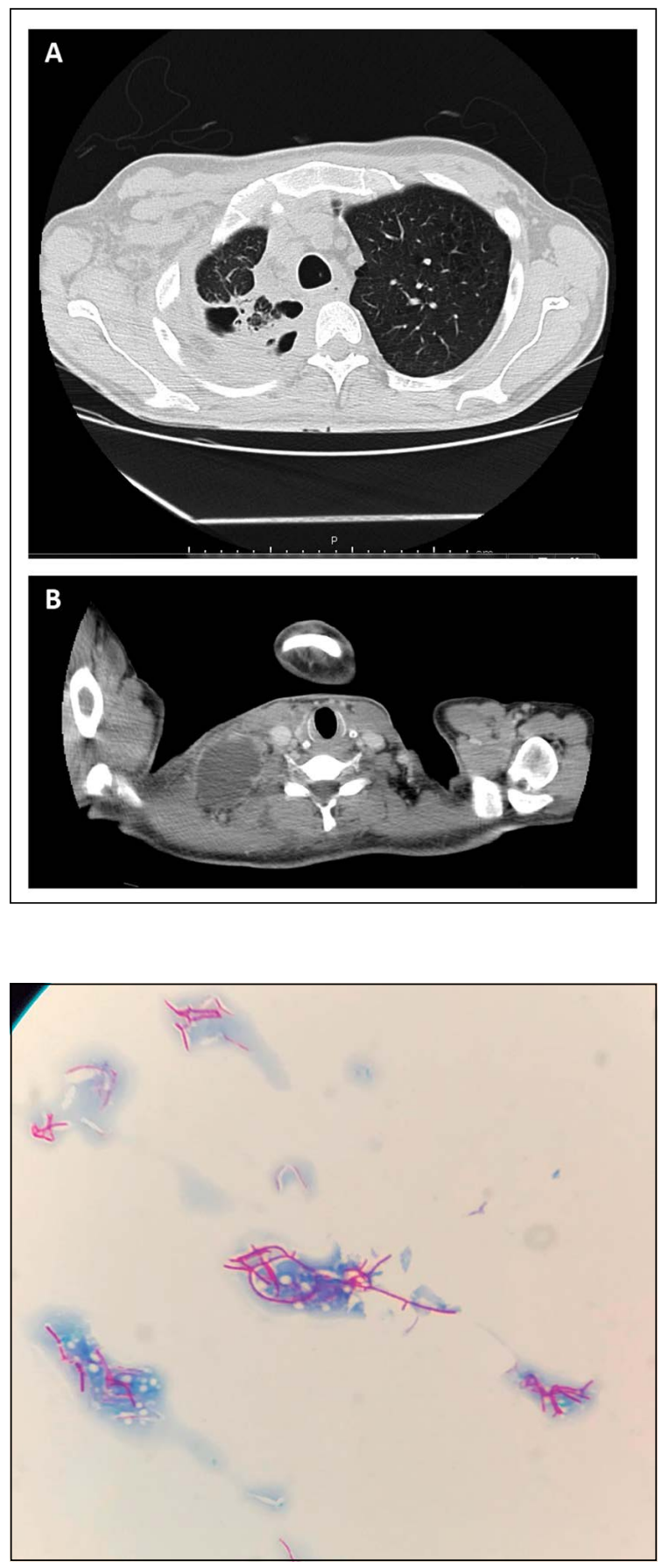

progresiva, a lo que se agregó un aumento de volumen cervical y torácico derechos. La ecografía cervical de tejidos blandos demostró un proceso inflamatorio con enfisema de la pared torácica paramediana derecha y la presencia de una masa adenopática necrótica supraclavicular derecha.

Reingresó al hospital para estudio, destacando hemocultivos automatizados y para micobacterias negativos. Se realizó una tomografía axial computarizada (TC) de cuello, tórax, abdomen y pelvis que mostró una consolidación excavada pulmonar en el lóbulo superior derecho asociado a múltiples adenopatías mediastínicas, algunas de ellas con centro hipodenso. Además de la presencia de una masa de origen ganglionar, con un centro necrótico en la región cervical basal derecha y axilar ipsilateral (Figura 3).

Fue sometido a una nueva fibrobroncoscopía estudiándose con baciloscopías, RPC para tuberculosis Xpert MTB/RIF, cultivo corriente y RPC para Pneumocystis jirovecii, todos negativos.

Se realizó una punción y drenaje del absceso cervical, con resultados de baciloscopía negativa y cultivo en medio líquido (MGIT) y sólido negativos. En el cultivo en agar sangre cordero 5\% (bioMérieux) de la colección, al quinto día de incubación, se desarrollaron colonias blanco-amarillentas, cuya tinción de Gram mostró bacilos grampositivos y en la tinción de Kinyoun resultaron ácido resistentes (Figuras 4 y 5). La identificación a nivel de especie se realizó mediante espectrometría de masas

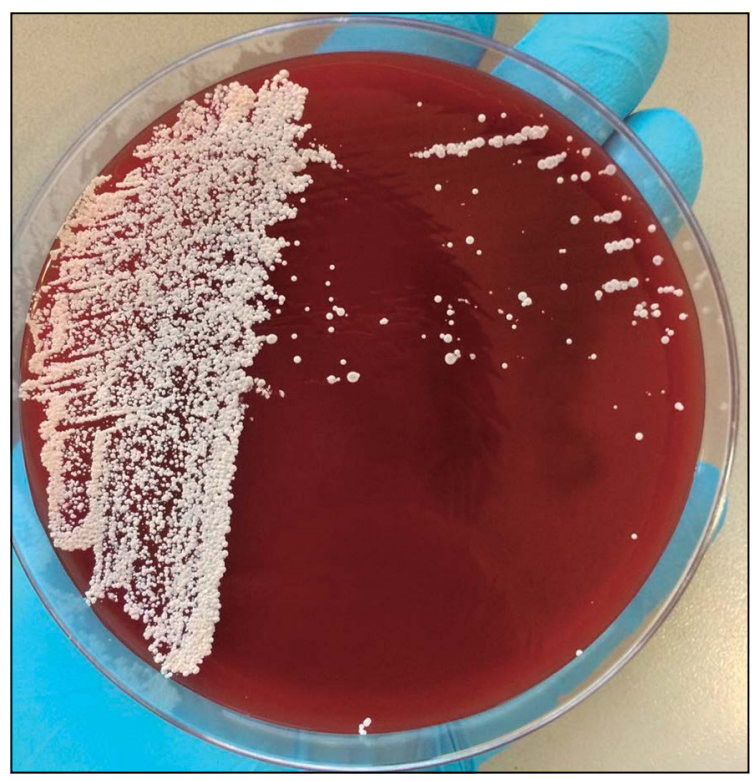

Figura 5. Cultivo en agar sangre de Nocardia cyriacigeorgica a los 5 días de incubación donde se observan colonias blanquecinas y secas (Foto original de Laboratorio Microbiología Hospital San Juan de Dios). 
MALDI-TOF (VITEK ${ }^{\circledR}$ MS, bioMérieux) con extracción previa con ácido fórmico, con un nivel de confianza de $99,9 \%$.

La confirmación se realizó mediante secuenciación del gen 16S rRNA, resultando una Nocardia cyriacigeorgica. El estudio de susceptibilidad por dilución en agar demostró que la cepa era sensible a amikacina, cefepime, cotrimoxazol, cloranfenicol e imipenem y resistente a cefotaxima y ciprofloxacina, según los puntos de corte de Clinical and Laboratory Standards Institute (CLSI, por sus siglas en inglés).

Recibió terapia con cotrimoxazol intravenoso (15 $\mathrm{mg} / \mathrm{kg} /$ día de trimetoprim) asociado a imipenem IV 500 $\mathrm{mg}$, cada $6 \mathrm{~h}$ por 14 días. Posteriormente, cotrimoxazol $1600 \mathrm{mg}$ cada $12 \mathrm{~h}$, vía oral, con buena respuesta clínica, presentando una regresión de la masa supraclavicular y mejoría de su condición general.

Al alta hospitalaria acudió a dos controles médicos posteriores; sin embargo, volvió a abandonar la TARV, sin haber logrado al momento del último control un alza de linfocitos CD4 ni CV indetectable.

\section{Discusión}

El género Nocardia se encuentra distribuido en todo el mundo, siendo ubicuo en el suelo y el agua. La transmisión proviene principalmente de la inhalación de esporas o mediante inoculación directa ${ }^{2}$.

Debido a la amplia distribución geográfica de las diferentes especies y a patrones de susceptibilidad diversos, la identificación a nivel de especie y el estudio de susceptibilidad se hace imprescindible ${ }^{3}$.

En el diagnóstico microbiológico se utilizan la tinción de Gram, que muestra bacilos gram positivos ramificados y la tinción de Kinyoun que es positiva para bacilos ramificados ácido alcohol parcialmente resistentes. Nocardia spp. crece en la mayoría de los medios no selectivos - como agar sangre de cordero, agar chocolate y Löwenstein-Jensen. Las colonias clásicamente se describen blanquecino-amarillentas con aspecto de tiza, secas y adherentes a la superficie del agar ${ }^{9,10}$; requieren un mínimo de 48 a 72 h de incubación para hacerse evidentes, lo que puede demorar dos a 14 días; por consiguiente, las placas no debiesen ser eliminadas antes de ese tiempo. Debido a esto, es imprescindible que se informe al laboratorio el antecedente clínico de inmunosupresión y la sospecha del diagnóstico.

La identificación por métodos convencionales fenotípicos tiene poco poder discriminatorio, es lenta $\mathrm{y}$ demandante, y no se recomienda en la actualidad. Sin embargo, las características macroscópicas de la colonia, debieran hacer sospechar Nocardia spp., con la consiguiente derivación a un laboratorio de referencia.
La identificación a nivel de especie, puede realizarse mediante espectrometría de masas (MALDI-TOF) o por métodos moleculares, mediante secuenciación del gen 16S rRNA. Sin embargo, en algunos casos es necesario incorporar otros genes para alcanzar la discriminación a nivel de especie (hsp65, rpoB o gyrB $)^{11}$. Eventualmente, pueden incluso ser necesarios análisis de tipificación multilocus de secuencias ${ }^{3,11}$.

El tratamiento incluye la cirugía en caso de abscesos o colecciones, disminuir la inmunosupresión cuando sea posible y el uso de antibacterianos por tiempo prolongado. Debido a reportes de alta resistencia a sulfas, se sugiere la terapia combinada, habitualmente con cotrimoxazol asociado a un carbapenémico, hasta estudio el susceptibilidad.

En relación a la sensibilidad antimicrobiana de las distintas especies, una revisión de 765 aislados de Nocardia en E. U. A entre los años 1995 al 2004, mostró que las especies más frecuentemente encontradas fueron $N$. nova (28\%), N. brasiliensis (14\%), N. farcinica $(14 \%) \mathrm{y}$ $N$. cyriacigeorgica $(13 \%)$. La resistencia a cotrimoxazol fue variable según la especie, con $20 \%$ de resistencia para $N$. brasiliensis, hasta $80 \%$ en $N$. farcinica ${ }^{12}$. Nocardia cyriacigeorgica presentó una susceptibilidad a cotrimoxazol de $82 \%$.

En Chile no contamos con datos locales de resistencia; sin embargo, en un caso clínico publicado de infección por $N$. farcinica, se describió resistencia a cotrimoxazol, cefotaxima y cefepime ${ }^{13}$.

Desde su descripción, $N$. cyriacigeorgica ha sido descrita como causante de infecciones clínicas en Europa, Asia y América. Al igual que las otras especies, se asocia principalmente a cuadros pulmonares; sin embargo, existen reportes de casos de bacteriemia y sepsis, infecciones del sistema nervioso central, piel, tejidos blandos $\mathrm{y}$ huesos ${ }^{14-17}$.

La susceptibilidad de $N$. cyriacigeorgica es variable. En un estudio realizado en España en 283 cepas se observó un 4,2\% de resistencia a cotrimoxazol y $2,8 \%$ a imipenem 6 . Por otro lado, en una revisión de los CDC, de 101 cepas de esta especie estudiadas, se observó una mayor proporción de resistencia ( $22 \%$ a cotrimoxazol y $23 \%$ imipenem $)^{12}$.

Nuestro paciente compartía parte del patrón de susceptibilidad que está descrito en especies de $N$. cyriacigeorgica pertenecientes al tipo $\mathrm{VI}^{18}$ (susceptibilidad a cotrimoxazol, imipenem, amikacina y linezolid; y resistencia a ampicilina, claritromicina y ciprofloxacina); esto permitió un tratamiento de mantención oral posterior con cotrimoxazol.

Como conclusión, la infección por Nocardia debe estar dentro de los diagnósticos diferenciales en pacientes inmunocomprometidos con compromiso pulmonar. Debe hacerse el esfuerzo en realizar un estudio microbiológico 
completo, idealmente con secuenciación genética para llegar al diagnóstico de especie.

Se debe informar al laboratorio de microbiología el antecedente de inmunosupresión y solicitar dirigidamente cultivo de Norcadia spp. para prolongar el tiempo de incubación.

El estudio de susceptibilidad es imprescindible para guiar la terapia antimicrobiana ajustada. En el documento M24 de CLSI se describe la metodología e interpretación de acuerdo a la $\mathrm{CIM}^{19}$.

\section{Referencias bibliográficas}

1.- Saubolle M A, Sussland D. Nocardiosis: review of clinical and laboratory experience. J Clin Microbiol 2003; 41: 4497-501. doi: 10.1128/ jcm.41.10.4497-4501.2003.

2.- Wilson JW. Nocardiosis: Updates and clinical overview. Mayo Clin Proc 2012; 87: 403-7. doi: 10.1016/j.mayocp.2011.11.016.

3.- Conville P S, Brown-Elliot B A, Smith T, Zelazni A M, The complexities of Nocardia taxonomy and identification. J Clin Microbiol 2017; 56: e01419-17. doi: 10.1128/JCM.0141917.

4.- Haussaire D, Fournier PE, Djiguiba K, Moala $\mathrm{V}$, Legris T, Purgus R, et al. Nocardiosis in the south of France over a 10-years period, 20042014. Int J Infect Dis 2017; 57: 13-20. doi: 10.1016/j.ijid.2017.01.005.

5.- Schlaberg R, Huard R.C. and Della-Latta P. Nocardia cyriacigeorgica, an emerging pathogen in the United States. J Clin Microbiol 2008; 46: 265-73. doi: 10.1128/JCM.00937-07.

6.- Valdezate S, Garrido N, Carrasco G, MedinaPascual M J, Villalón P, Navarro A M, et al. Epidemiology and susceptibility to antimicrobial agents of the main Nocardia species in Spain. J Antimicrob Chemother 2017; 72: 754-61. doi: 10.1093/jac/dkw489.

7.- $\quad$ Yu S, Wang J, Fang Q, Zhang J, Yan F. Specific clinical manifestations of Nocardia: A case report and literature review. Exp Ther Med 2016; 12: 2021-6. doi: 10.3892/etm.2016.3571.
8.- Khadka P, Basnet RB, Rijal BP, Bahadur J. Pulmonary nocardiosis masquerading renascence of tuberculosis in an immunocompetent host: a case report from Nepal. BMC Res Notes 2018; 11: 488. doi: 10.1186/s13104-018-3604-2.

9.- Brown-Elliott B A, Brown J M, Conville P $\mathrm{S}$, Wallace R J Jr. Clinical and laboratory features of the Nocardia spp. based on current molecular taxonomy. Clin Microbiol Rev 2006; 259-82. doi: 10.1128/CMR.19.2.259-282.2006.

10.- McNeil M M, Brown J M. The medically important aerobic actinomycetes: epidemiology and microbiology. Clin Microbiol Rev 1994; 7: 357-417. doi: 10.1128/cmr.7.3.357.

11.- Xiao M, Pang L, Chen SC-A, Fan X, Zhang $\mathrm{L}$, Li H-X, et al. Accurate identification of common pathogenic Nocardia species: evaluation of a Multilocus Sequence Analysis Platform and Matrix-Assisted Laser Desorption Ionization-Time of Flight Mass Spectrometry. PLoS One 2016; 11: e0147487. doi: 10.1371/ journal.pone.0147487.

12.- Uhde K, Pathak S, McCullum I, Jannat-Khah D P, Shadomy S V, Dykewicz CA, et al. Antimicrobial-resistant Nocardia isolates, United States, 1995-2004. Clin Infect Dis 2010; 51: 1445-8. doi: 10.1086/657399.

13.- Gutiérrez C, López T, Rivera G, León E, Castillo C, Ajenjo M C. Infección por Nocardia farcinica con resistencia a sulfas en paciente inmunocomprometida. XXVIII Congreso Chileno de Infectología,
Coquimbo, Chile. Noviembre, 2011. Libro de resúmenes P-55. Rev Chilena Infectol 2011; 28 (S2): S88.

14.- Elsayed S, Kealey A, Coffin C S, Read R, Megran D, Zhang K. Nocardia cyriacigeorgica septicemia. J Clin Microbiol 2006; 44: 280-2. doi: 10.1128/JCM.44.1.280-282.2006.

15.- Barnaud G, Deschamps C, Manceron V, Mortier E, Laurent F, Bert F, et al. Brain abscess caused by Nocardia cyriacigeorgica in a patient with human immunodeficiency virus infection. J Clin Microbiol 2005; 43: 4895-7. doi: 10.1128/JCM.43.9.4895-4897.2005.

16.- Khorshidi M, Navid S, Azadi D, Shokri D, Shojaei H, et al. A case report of brain abscess caused by Nocardia cyriacigeorgica in a diabetic patient. JMM Case Rep 2018; 5. e005133. doi: 10.1099/jmmcr.0.005133.

17.- Raszka D, Popelka S Jr, Hert J, Jahoda D, Landor I, Vavrik P. Rare case of osteomyelitis of tibial shaft caused by Nocardia cyriacigeorgica. Folia Microbiol (Praha) 2018; 63: 525-32. doi: 10.1007/s12223-018-0589-0.

18.- Brown-Elliott B A, Biehle J, Conville P S, Wallace R Jr. Current status of Nocardia taxonomy and recommended identification methods. Clin Microbiol Newsletter 2015; 37: 25-32. doi: 10.1016/j. clinmicnews.2015.01.007.

19.- M24 CLSI. "Susceptibility Testing of Mycobacteria, Nocardia spp., and Other Aerobic Actinomycetes", 3rd Edition. November 28, 2018. 